Title: Does expecting nociceptive stimuli in non-adjacent skin areas have an analgesic effect on stimuli presented between them?

\title{
Running title: Modulation of pain via expectation of its location
}

TR Stanton, ${ }^{1,2}$ H Gilpin, ${ }^{1,3}$ E Reid, ${ }^{1}$ F Mancini, ${ }^{4}$ C Spence, ${ }^{5}$ \& GL Moseley ${ }^{1,2}$

${ }^{1}$ The Sansom Institute for Health Research, The University of South Australia, \& PainAdelaide, Adelaide, 5001, Australia

${ }^{2}$ Neuroscience Research Australia, Sydney, 2031, Australia

${ }^{3}$ School of Psychology, The University of Nottingham, Nottingham, NG7 2RD, UK

${ }^{4}$ Neuroscience, Physiology and Pharmacology Department, University College London, London, WC1E 6BT, UK \& Institute of Cognitive Neuroscience, University College London, London, WC1N 3AR, UK

${ }^{5}$ Crossmodal Research Laboratory, Department of Experimental Psychology, University of Oxford, Oxford, UK

\section{Corresponding Author:}

Tasha R Stanton

The University of South Australia

School of Health Sciences - CEA-14

GPO Box 2471

Adelaide, South Australia 5001

Phone: +618 83022090

Fax: +618 83022853 
Email: Tasha.stanton@unisa.edu.au

Submission category: Original article

Disclosures: This study was supported by National Health and Medical Research Council Project Grant (ID 1008017). TRS supported by Canadian Institute for Health Research Postdoctoral Training Fellowship (ID223354) and National Health \& Medical Research Council Early Career Fellowship (ID 1054041). GLM supported by a National Health and Medical Research Council Research Fellowship (ID1061279). FM is supported by a Wellcome Trust Project grant (094863/Z/10/Z). TRS received travel and accommodation support from Eli Lilly Ltd. for a Western Canada speaker's tour (September 2014); this was unrelated to the present topic.

\section{What's already known about this topic?}

- Spatial attention and expectation can modulate pain in a regional manner.

\section{What does this study add?}

- The spatial acuity with which expectancy can modulate pain is more precise than previously thought.

- The effect of expectancy on pain is dependent on both the expected, and actual, location of nociceptive stimulation.

- A proximal-distal pain gradient exists on the forearm: identical nociceptive stimuli are perceived as less painful when delivered distally (versus proximally).

Number of text pages: 28

Number of Figures: 2

Number of Tables: 3

Word count: Abstract (250 words); Introduction (500 words); Discussion (1496 words) 


\begin{abstract}
:
Background: The spatial precision of expectancy effects on pain is unclear. We hypothesised that expecting nociceptive stimuli at particular skin areas would have an analgesic effect on nociceptive stimuli presented between them (middle zone).
\end{abstract}

Methods: Laser stimuli (evoking pin-prick pain), were delivered to three discrete skin zones on the forearm, under two conditions. During 'Localisation', participants' expectation of stimuli was spatially-divided between two locations (expected stimuli in only the outer two skin zones): pain intensity and stimulus location were judged. During 'No-localisation' (control), the participants had no expectation concerning stimulus location; only pain intensity was rated. Additional experiments assessed the importance of the actual location on the forearm by: shifting all skin zones proximally towards the elbow (control for joint proximity, Experiment 2); adding a fourth zone distally (control for interaction between joint proximity and enhanced distal inhibition, Experiment 3).

Results: All experiments demonstrated spatially-specific pain modulation, but only Experiment 2 (near elbow) supported our hypothesis: middle zone pain intensity was significantly lower $(\mathrm{p}=0.02)$ during Localisation than No-localisation. Experiment 1 (near wrist) found reduced pain intensity during Localisation only for the distal zone $(\mathrm{p}=0.04)$. Experiment 3 confirmed this effect: reduced pain during Localisation occurred only for the most distal zone $(\mathrm{p}=0.046)$.

Conclusion: Expecting a painful stimulus in non-adjacent skin areas has spatially-specific effects on pain modulation, but this reflects an interaction between the expected location of stimulation and their actual location. This suggests a more complex connection between 
somatotopic maps and nociceptive modulation than previously thought; several distinct mechanisms likely contribute. 


\section{Introduction}

The detection and modulation of sensory information is critical in generating specific sensations (Koyama et al., 2005) and optimising our response to external stimuli (Morgan and Whitney, 1996). This dynamic modulation of incoming somatosensory information occurs at many levels, including the dorsal horn (Hayes et al., 1981), brainstem, thalamus, and cortex (Faggin et al., 1997).

Spatial attention modulates pain (Johnston et al., 2012; Legrain et al., 2002; Quevedo and Coghill, 2007), although its role is complex. Event-related potentials (ERPs) elicited by nociceptive stimuli are enhanced in magnitude when delivered to an attended location versus an unattended location (Legrain et al., 2002), but, attending to a specific body part when expecting an intensely painful stimulus reduces the stimulus-evoked pain (Johnston et al., 2012). Furthermore, dividing spatial attention, by delivering two simultaneous nociceptive stimuli to non-adjacent spatial locations within a body segment, is known to reduce evoked pain (versus pain evoked by single nociceptive stimuli at either location; Quevedo and Coghill, 2007).

Analgesia during divided spatial attention is hypothesised, in part, to be mediated by changes in receptive field (RF) properties of dorsal horn neurons (Quevedo and Coghill, 2007). Attention can alter the spatial properties of medullary dorsal horn neuron RFs (Hayes et al., 1981). When dividing attention between two non-adjacent areas, psychophysical findings suggest that nociceptive RFs become smaller, shifted, and more 'focussed' on the two attended areas (Quevedo and Coghill, 2007). One would hence predict that the area of skin between these two attended areas would become comparatively 'out of focus', thus creating a ‘silent zone’(Quevedo and Coghill, 2007) where sensitivity to nociceptive stimuli may be reduced. 
We interrogated this idea by manipulating expectancy: would expecting nociceptive stimuli in particular skin areas modulate the stimulus-evoked pain between them? We compared two conditions: during 'Localisation', participants expected stimuli in two spatially non-adjacent areas; their task was to localise stimuli and rate pain intensity. During 'No localisation' (control), the participants had no expectation concerning stimulus location, rating only pain intensity. We hypothesised that when nociceptive stimuli are expected in two non-adjacent skin zones, stimuli delivered to an 'unexpected' middle zone ('unfocussed' area) would be less painful during Localisation (versus No-localisation) but that this analgesia would not occur for either 'expected' skin zone. This effect would be predicted by the 'focussed' hypothesis of dynamic modulation of dorsal horn RFs, but not by more general attention effects (e.g., distraction via a cognitive task; Bantick et al., 2002; McCaul and Haugtvedt, 1982). It would also be more precise than general spatial attention effects that are evident in tactile processing during cued attention to a body segment or region of space (Van Damme et al., 2009).

Given that sensitivity to cutaneous stimuli differs based on proximity to a joint (Cholewiak and Collins, 2003), and that a proximal-to-distal gradient in nociceptor afferent density exists in the arm (McArthur et al., 1998), our aim was to determine whether the actual location of delivered nociceptive stimuli would influence this expectation-induced effect. We completed three separate experiments that manipulated the location of stimuli delivered to the forearm.

\section{Experiment 1}

In Experiment 1, we tested whether expecting to receive nociceptive stimuli in two discrete areas would result in an analgesic effect to nociceptive stimuli delivered in the skin area between them. In this experiment, the stimulation areas were situated close to the wrist. 


\section{Methods}

\section{Study design}

We conducted a randomised, repeated measures behavioural experiment with healthy volunteers. The experiment involved two conditions that were undertaken in random, counterbalanced order and separated by at least 45 minutes.

\section{Participants}

A convenience sample of 18 right-handed, healthy volunteers (12 females) without current pain or a history of chronic pain participated (see Table 1 for participant characteristics). The participants were recruited via poster advertisements placed within the Institution and on community notice boards and also via word of mouth. All of the participants were required to be naïve to pain research using laser stimuli. Additionally, the participants were required to have normal sensation and to be free of any neurological conditions that might affect sensation or the processing of noxious stimuli (e.g., stroke, brain injury, neuropathy), as well as free of any skin condition that could compromise the safety of laser application (open wounds on the arm, psoriasis, or severe eczema). Those who were currently taking any medications that might alter pain (e.g., paracetamol, aspirin, non-steroidal antiinflammatories) or who indicated on questioning that they had consumed alcohol in the eight hours prior to testing, were excluded from the study. All of the participants provided their written informed consent and the study was approved by the Institutional Ethics Committee and was in accordance with the Helsinki Declaration of 1975, as revised in 1983.

\section{Nociceptive stimuli}

The participants were seated with both of their arms resting on a table, with their palms facing downwards, and the dorsal aspect of the forearm horizontal (see Figure 1). 
Nociceptive stimuli were delivered to the dorsal aspect of the right forearm using an infrared neodymium yttrium aluminium perovskite (Nd:YAP) $1034 \mu \mathrm{m}$ laser (Elen, Italy). At this wavelength, laser pulses directly activate A-delta and C-fiber nociceptive terminals in the superficial layers of the skin (Iannetti et al., 2006). We used a pulse duration of 5ms, a spot diameter of approximately $7 \mathrm{~mm}$ and an inter-stimulus interval of 30sec. The laser optics were secured above the forearm using a pinch stand, such that there was no contact with the forearm and so that the distance between the skin and the laser was standardised. The palm and wrists of both hands rested on small blocks to ensure that the entire skin surface of the forearm was perpendicular to (and the same distance from) the laser beam.

Participants’ pinprick sensation threshold - the lowest laser energy at which participants felt a clear pinprick sensation - was determined. The participants were then introduced to the 101 point numerical rating scale (NRS) for pain, anchored with zero $=$ no sensation of painful pinprick at all, and $100=$ the most painful pinprick sensation imaginable. This measure, herein called 'Pain', was the primary outcome variable. The laser pulse energy was increased until participants rated the evoked pain as 20/100 or greater, at all stimulation locations tested on the forearm. This energy level was used for all subsequent laser pulses and all participants reported experiencing a clear painful 'pin-prick' sensation. The average energy used for all stimulations was $3.9 \mathrm{~J} \pm 0.5 \mathrm{~J}$. Because a change in baseline skin temperature will change the nociceptive barrage evoked by laser stimulation, we assessed the temperature of the arm before and after each condition, using a thermal imaging camera (FLIR technology, sc620) and FLIR software analysis (FLIR systems 2009; sensitivity <40 milli-Kelvin degrees; accuracy $<2 \%$ over the physiological temperature range). Change in skin temperature within each skin zone (i.e., temperature post-condition minus temperature pre-condition - ' $\Delta \mathrm{T}$ ') was analysed.

\section{Experimental set-up}


During testing, the participant's view of their right arm was occluded using an opaque cloth, suspended from a ceiling bar. In this way, the participants could only use information from A-delta and C-fibre input to make their judgements about stimulus location (see below for 'Localisation' condition explanation). A mirror was situated in line with the opaque cloth and at such an orientation that the reflected image of the left arm appeared to participants to be their right arm (Egsgaard et al., 2011) (Fig. 1). The participant’s left and right hands were positioned such that they were spatially congruent both with each other and in terms of their distance from the body midline. The mirror was used to: (i) ensure that the participants continued to attend to their right arm during testing; (ii) provide non-informative vision of the right arm; a strategy shown to improve spatial resolution for somatosensory stimuli (Kennett et al., 2001). Because our testings conditions differed based on whether or not participants provided localisation judgements for nociceptive stimuli on the arm (see below), it was of interest to heighten the ability to localise stimuli.

\section{Testing procedure and conditions}

Laser stimuli were delivered within three zones on the participant's hidden forearm (proximal, middle, and distal zone). Each zone was 2cm long, and was separated by approximately 2cm (Fig. 2). The inside borders of the two outside zones (proximal and distal) were separated by $5.8 \mathrm{~cm}$; this corresponds to the average two-point discrimination threshold for laser stimuli (Morch et al., 2010). This separation was chosen because it would suggest that we were stimulating at least three discrete nociceptive receptive fields on the forearm. The distal zone (distal border) was located $5 \mathrm{~cm}$ proximal to the wrist crease, as identified by palpating and marking the ulnar styloid process.

There were two testing conditions: ‘Localisation’ and ‘No-localisation'. Conditions were undertaken in a randomised and counterbalanced order. In both conditions, the participants 
reported pain (0-100 NRS) directly after each stimulus. During No-localisation, participants had no expectation of where stimuli would be provided and rated only pain intensity. During Localisation, in addition to rating pain, the participants were asked to judge in which area of skin (proximal or distal zone) the stimulus had occurred. The participants were instructed: "You will be receiving laser stimuli on your arm. These stimuli will be given to this area $<$ point to area $>$, called the proximal zone, and this area $<$ point to area $>$, the distal zone. For each laser stimulus, your task is to tell us in which zone, proximal or distal, you received the stimulus.” The borders of the proximal and distal zones were drawn on both forearms (Fig. 2) such that participants could clearly see the zones on the mirror-reflected image of the left arm.

In both conditions, the participants received a total of 20 laser stimuli at each of the proximal and distal zones, and received 10 laser stimuli at the unexpected middle zone. The order of stimulation was randomised and counterbalanced, and there were at least two 'outside' stimuli (delivered to proximal/distal zones) between each stimulus delivered to the middle zone. A smaller number of pulses were chosen for the middle zone so as to minimise the likelihood that they would be detected as occurring outside of the expected zones. The sequence of stimulation varied between participants, but was identical for both conditions within participants. To prevent over-heating the skin, nociceptor sensitisation and fatigue, the precise location of laser stimulation was adjusted slightly between stimuli. However, all stimuli occurred within the allotted zone.

For the Localisation condition, participant responses for stimuli that were delivered to the proximal or distal zones were coded as correct or incorrect, but no feedback was provided. Participant responses for stimuli delivered to the middle zone were coded by the zone that was named, for example - for a middle zone stimulus judged by the participant to occur in 
the proximal zone, it was coded: “Proximal”. Responses were analysed to evaluate whether any systematic perceptual bias towards one zone or another occurred.

Following completion of the Localisation condition, participants were instructed to remove their arm from behind the vertically positioned opaque cloth. Before they could see their arm, an opaque screen was held horizontally over the participant's right arm, so that vision of the arm was occluded, and participants indicated with their left hand where, on the right arm, they felt that the majority of the stimuli had been delivered ("Please point to the main areas on your [right] arm where you felt that you received the majority of the stimuli”). The researcher noted whether the participants were accurate, or pointed proximally or distally to the actual areas stimulated. Vision was occluded because laser stimuli can leave red marks on the skin: we were interested in a perceptual, not visually-mediated, judgement of stimulus location. Last, a manipulation check was undertaken by asking the participants whether they had felt stimuli in any areas of their arm other than the proximal or distal zones.

\section{Analysis}

We performed a 2 × 3 repeated measures analysis of variance (ANOVA) on average pain with two within-participants factors: Condition (Localisation or No-localisation) and Zone (Proximal, Middle, or Distal). Where appropriate, post-hoc paired t-tests were used for specific comparisons. We were powered to detect a moderate interaction effect $(\mathrm{f}=0.35$, partial $\eta^{2}=0.11$ ) based on a $2 \times 3$ repeated measures ANOVA, using a standard deviation for evoked pain of 7 points on a 101 point NRS (Iannetti et al., 2008), a conservative correlation of 0.5 between measures, $80 \%$ power and alpha $=0.05$ ( $n=16$ participants); calculated using G*Power 3.1.5 (Faul et al., 2007). Based on these parameters, this sample size provides sufficient power (>0.80) for post-hoc paired comparisons to detect a mean difference of 4 
points (Van Ryckeghem et al., 2011) $\left(S_{\text {diff }}=10\right)$ on a 101 point NRS (i.e., $\left.d=0.40\right)$. Partial $\eta^{2}$ and Cohen’s d (dependent samples; Faul et al., 2007) are provided as effect size measures.

Higher skin temperature will lead to a higher post-stimulus temperature of the skin and, presumably, differential activation of nociceptors. On the grounds that cognitive mechanisms can modulate skin temperature in discrete body parts (Moseley et al., 2008), we set out to identify whether skin temperature changed in a zone-specific or condition-specific manner. A 2 x 3 repeated measures ANOVA (Condition x Zone) was undertaken on $\Delta \mathrm{T}$. If there were any Zone, Condition, or Zone $\mathrm{x}$ Condition effects on $\Delta \mathrm{T}$, then $\Delta \mathrm{T}$ was entered as a covariate in the ANOVAs on Pain.

Performance in the Localisation task was calculated as the average error per participant. Perceptual data were presented using descriptive statistics.

\section{Results}

A total of 22 participants were screened to participate; of these, 4 were left handed and thus were excluded. Table 1 provides participant information.

\section{Pain ratings}

The ANOVA revealed no differences in pain ratings between Conditions $\left(F_{1,17}=2.4, p=0.14\right.$, partial $\left.\eta^{2}=0.13\right)$, but a main effect of Zone $\left(F_{2,34}=17.1, p<0.001\right.$, partial $\left.\eta^{2}=0.50\right)$. Pain was higher for stimuli delivered to the proximal zone (21.5, 95\%CI 17.1 to 25.9 ) than for stimuli delivered to the middle (17.6, 95\%CI 14.0 to $21.2 ; \mathrm{p}<0.01)$ and distal zones $(16.9,95 \% \mathrm{CI}$ 13.3 to 20.4 , $\mathrm{p}<0.001$ ). There was no difference between middle and distal zone pain $(p=0.28)$. Post hoc tests, considering the effect of Zone separately for each condition, support this main effect (see results S1). There was also a Condition $\mathrm{x}$ Zone interaction $\left(\mathrm{F}_{2,34}=4.4\right.$, 
$\mathrm{p}=0.02$, partial $\eta^{2}=0.21$ ). Post-hoc tests revealed that pain ratings were lower during Localisation than during No-localisation for stimuli delivered to the distal zone $(\mathrm{d}=0.53$, $\mathrm{p}=0.04)$ but not for stimuli delivered to the other zones $(\mathrm{d}=0.45, \mathrm{p}=0.07$ for middle and $d=0.19, p=0.43$ for proximal; see Fig. 2 and Table 1 ). That is, the task of localising stimuli reduced the pain evoked by stimuli delivered to the distal zone.

Visual inspection of pain ratings during No-localisation and Localisation in each individual revealed that ten out of the 18 participants had less pain for distal zone stimuli during Localisation than they did during No-localisation, consistent with the group-level statistical findings. FigureS1 provides the pain ratings for each zone and for each condition over time.

The order in which the participants undertook the two conditions did not affect pain ratings. Specifically, when controlling for Task order, there was no effect of Task order $\left(F_{1,16}=0.02\right.$, $\mathrm{p}=0.89)$, but the main effect of Zone $\left(\mathrm{F}_{2,32}=16.6, \mathrm{p}<0.001\right)$, and the Zone $\mathrm{x}$ Condition interaction $\left(F_{2,32}=4.3, p=0.02\right)$, remained significant. Similar to the main analysis, there was no effect of Condition $\left(\mathrm{F}_{1,16}=2.5, \mathrm{p}=0.14\right)$, and all other interactions were non-significant (Zone $\mathrm{x}$ Task order, $\mathrm{p}=0.64$; Condition $\mathrm{x}$ Task order, $\mathrm{p}=0.25$; Zone $\mathrm{x}$ Condition $\mathrm{x}$ Task order, $\mathrm{p}=0.47)$.

\section{Skin temperature}

The change in temperature ( $\Delta \mathrm{T}$; see Table 2$)$ was different between zones and conditions. The temperature change was largest in the proximal zone and temperature change during Nolocalisation was larger than during Localisation. Specifically, there was a main effect of Zone $\left(F_{2,34}=3.7, p=0.03\right.$; proximal $>$ middle, $p<0.01$, no other differences) and a main effect of Condition $\left(\mathrm{F}_{1,17}=4.7, \mathrm{p}=0.045\right.$; No-localisation $\Delta \mathrm{T}:-0.42^{\circ}$ Celsius, Localisation $\Delta \mathrm{T}:-0.01^{\circ}$ Celsius). There was no Condition $\mathrm{x}$ Zone interaction $\left(\mathrm{F}_{2,34}=0.12, \mathrm{p}=0.88\right)$. Due to these differences, $\Delta \mathrm{T}$ was added as a covariate to the pain analysis. When controlling for $\Delta \mathrm{T}$, the 
pain results were unaltered. There was no effect of any $\Delta T$ at any Zone/Condition ( $p>0.17$ for all $)$ and no effect of Condition $\left(F_{1,11}=2.2, p=0.17\right)$. The main effect of Zone $\left(F_{2,22}=5.8\right.$, $\mathrm{p}<0.01)$ and the Zone $\mathrm{x}$ Condition interaction $\left(\mathrm{F}_{2,22}=3.5, \mathrm{p}=0.047\right)$ remained significant. Only one other interaction was significant ( $\mathrm{p}>0.14$ for all others) and this was between Condition $\mathrm{x}$ Zone $\mathrm{x} \Delta \mathrm{T}$ in the distal zone during Localisation $\left(\mathrm{F}_{2,22}=7.4, \mathrm{p}<0.01\right)$. These analyses demonstrate that the pain-related results are not simply a reflection of changes in baseline skin temperature.

\section{Localisation performance}

The participants made few errors when attempting to localise the laser stimuli (mean \pm SD error rate $=6 \% \pm 4 \%$ for the 20 distal zone stimuli and $1 \% \pm 4 \%$ for the 20 proximal zone stimuli). Errors tended to decrease over time (Table 3). Participants attributed $66.1 \% \pm 20 \%$ of middle zone stimuli to the proximal zone and $33.9 \% \pm 20 \%$ to the distal zone. When asked to point to the areas of the arm where they felt that they had received the majority of the stimuli, most participants perceived the proximal and distal zones to be more distal than their actual location.

The manipulation check revealed that five participants thought that they had received stimuli between the proximal and distal zones. We did not statistically evaluate whether or not detecting stimuli outside of the zone was associated with different pain ratings, because it was not an a priori question; however, visual inspection of the data suggested that pain ratings were no different between these five participants and the others (FigureS2).

\section{Experiment 1 Discussion}

Our findings were contrary to our hypothesis; pain was reduced in the distal zone during Localisation but not in the middle zone as hypothesised. No main effect of condition 
(Localisation versus No-localisation) suggests against a general effect of task load (Romero et al., 2013) during the Localisation condition. That is, despite Localisation being a dual-task paradigm (localisation + rating pain intensity) and No-localisation being a single task paradigm (only rating pain intensity), this additional cognitive load during Localisation did not result in analgesia for all zones. Thus while the effect was not as hypothesised, the results suggest that the pain modulation is spatially-specific.

Distal zone analgesia when expecting nociceptive stimuli in that zone (but no proximal zone analgesia despite identical expectations) suggests that proximity to a joint may contribute to this analgesic effect (Cholewiak and Collins, 2003). The main effect of Zone provides evidence for a proximal-to-distal pain gradient to laser stimuli on the forearm and raises the possibility that, if focussing of RFs, via the Localisation task, occurs in areas with lower nociceptive density, then distal stimuli may experience increased inhibition. There is evidence from lower limb data that inhibitory effects on pain are stronger distally than proximally (Quevedo and Coghill, 2007). If this phenomenon were also to occur in the upper limb, it is possible that distal zone analgesia during Localisation, observed here, reflects just such an effect. Thus a question left unanswered is whether this spatially-specific analgesic effect of Localisation is modulated by the 'expected skin area's' proximity to a joint or by a distal-proximal gradient, independent of proximity to a joint, whereby distal locations will always experience the most inhibition. To answer this question, we undertook a second experiment.

\section{Experiment 2}

We tested whether moving the zones closer to the elbow would alter the effect of spatially dividing expectation. If proximity of nociceptive stimuli to a joint is the main driver of expectancy-induced analgesia in Experiment 1, then moving the zones towards the elbow 
should result in decreased pain scores during Localisation for only the most proximal zone (located closer to the elbow than the distal zone is to the wrist). Alternatively, if the results of Experiment 1 reflect a distal -proximal gradient of the inhibitory effects of cognitive manipulations, then we would not expect the proximal zone to give rise to reduced pain scores during Localisation compared with No-localisation, but would expect replication of Experiment 1.

\section{Method}

\section{Study design}

Randomised repeated measures experiment, involving two conditions separated by 45 minutes, with the order of conditions randomised and counterbalanced.

\section{Participants}

A convenience sample of 16 right-handed, healthy volunteers (5 female) participated. Eligibility criteria were identical to Experiment 1. All participants provided their written informed consent and the study was approved by the Institutional Ethics Committee and was in accordance with the Helsinki Declaration of 1975, as revised in 1983.

\section{Nociceptive stimuli and experimental procedure}

Experiment 2 replicated the protocol of Experiment 1, using identical nociceptive stimuli and methodology, except that the testing zones were located at the proximal instead of the distal end of the forearm. Specifically, the proximal zone (proximal border) was located $5 \mathrm{~cm}$ distal to the elbow crease (Fig. 2). Zone width and distances between zones were identical to those used in Experiment 1. In this way, the distal zone in Experiment 2 coincided approximately with the middle zone in Experiment 1. 


\section{Analysis}

An identical power analysis was used to inform recruitment for Experiment 2 (n=16 required). We performed a 2 x 3 repeated measures analysis of variance (ANOVA) on Pain with two within-participants factors: Condition (Localisation or No-localisation) and Zone (Proximal, Middle, or Distal). Where appropriate, post-hoc paired t-tests were used for specific comparisons. As per Experiment 1, $\Delta \mathrm{T}$ was added as a covariate into the pain analysis if there were any Zone, Condition, or Zone $\mathrm{x}$ Condition effects on $\Delta \mathrm{T}$. Localisation performance was summarised as per Experiment 1.

\section{Results}

A total of 18 participants were screened for inclusion; of these, one was left-handed and thus excluded and one participant declined to participate after initial testing (anxious about laser stimulation).

\section{Pain ratings}

Pain was lower during Localisation than No-localisation for stimuli delivered to the middle zone, which was consistent with our original hypothesis. Similar to Experiment 1, the same proximal-to-distal gradient in pain ratings was observed: the ANOVA revealed a significant main effect of Zone $\left(F_{2,30}=24.4, p<0.001\right.$, partial $\left.\eta^{2}=0.61\right)$. Proximal zone pain ratings (23.4, 95\%CI 17.1 to 29.7 ) were higher than middle (18.7, 95\%CI 11.3 to 26.0 ; $\mathrm{p}<0.01)$ and distal (16.2, 95\%CI, 9.0 to 23.4; $\mathrm{p}<0.001)$ zone pain ratings. The middle zone pain ratings were, in turn, higher than the distal pain ratings ( $\mathrm{p}<0.001$; Fig. 2 and Table 1). Post hoc tests, considering the effect of zone separately for each condition, support this main effect (see results $\mathrm{S1})$. There was no difference in pain ratings between Conditions $\left(\mathrm{F}_{1,15}=2.9, \mathrm{p}=0.12\right.$, partial $\left.\eta^{2}=0.16\right)$, but there was a Condition $\mathrm{x}$ Zone interaction $\left(\mathrm{F}_{2,30}=3.8, \mathrm{p}=0.04\right.$, partial $\eta^{2}$ 
$=0.20$ ), such that identical stimuli delivered to the middle zone were rated as less painful during Localisation than during No-localisation $(\mathrm{d}=0.63, \mathrm{p}=0.02)$. There were no differences in pain ratings between conditions in the proximal $(d=0.12, p=0.64)$ or distal zones $(d=0.18$, $\mathrm{p}=0.21)$

Visual inspection of the pain ratings in each individual revealed that 11 of 16 participants gave lower pain ratings in the middle zone during the Localisation than during Nolocalisation, which was consistent with the group-level statistical analysis. FigureS3 provides the pain ratings specific to zone and condition over time.

The results were unchanged when we controlled for task order (No-localisation first or Localisation first). That is, there was no effect of Task order $\left(\mathrm{F}_{1,14}=1.9, \mathrm{p}=0.19\right)$, and no effect of Condition $\left(\mathrm{F}_{1,14}=2.8, \mathrm{p}=0.11\right)$, but there remained a main effect of Zone $\left(\mathrm{F}_{2,28}=23.8\right.$, $\mathrm{p}<0.001)$, and a Zone $x$ Condition interaction $\left(\mathrm{F}_{2,28}=3.8, \mathrm{p}=0.03, \mathrm{p}>0.34\right.$ for all other tests $)$.

\section{Skin temperature}

There was no difference in $\Delta \mathrm{T}$ (see Table 2 ) based on Zone $\left(\mathrm{F}_{2,30}=2.1, \mathrm{p}=0.14\right)$ or Condition $\left(F_{1,15}=0.03, p=0.87\right)$, and there was no Zone $x$ Condition interaction $\left(F_{2,30}=0.04, p=0.29\right)$.

\section{Localisation performance}

Participants performed worse on the localisation task when stimuli were delivered to the distal zone (mean error \pm standard deviation: $16 \% \pm 7 \%$ ) than when they were delivered to the proximal zone $(4 \% \pm 5 \%)$. There was no change in localisation performance over time for either zone (Table 3). Participants attributed $59.0 \% \pm 24.0 \%$ of middle zone stimuli to the proximal zone and $40.6 \% \pm 23.5 \%$ to the distal zone.

When asked, five of the participants reported that they thought that they had received stimuli in the middle zone. Again, we did not analyse this because it was not an a priori question, but 
visual inspection of the data suggested that pain ratings were no different for these five than they were for the remainder of the participants (figure S4).

\section{Experiment 2 Discussion}

This experiment found evidence for our original hypothesis, that dividing expectation between two locations results in analgesia specific to nociceptive stimuli applied between these locations. The lack of analgesia in the proximal zone during Localisation suggests against proximity to any joint being the key feature in pain modulation. The lack of analgesia during Localisation in the distal zone, suggests against merely distal location (relative to the other zones) as a primary factor in expectancy-induced pain modulation. Once again, the lack of a main effect of Condition suggests that these effects are not general effects of task load (Romero et al., 2013) and supports the idea that expectancy-based pain modulation can be spatially precise. That Experiments 1 and 2 found different effects for pain modulation, despite identical methodology, suggests that there may be an interaction between the actual location of nociceptive stimuli application on the forearm and the task.

Overall, localisation errors were higher in Experiment 2 than in Experiment 1: task difficulty may have differed between experiments. Use of a two-choice paradigm raises the possibility that some participants utilized a yes/no response strategy and focussed on whether the stimulus was delivered to one particular zone or not. This strategy would certainly be easier to use in areas with lower overall localisation errors (Experiment 1). That mislocalisation was very low in the proximal zone in Experiment 1 would appear to be consistent with such a strategy focussed on the proximal zone. We did not attempt to identify such a strategy and have no way of determining it post-hoc. Additionally, while a proximal-to-distal pain gradient was found in both Experiments 1 and 2, it is possible that inhibitory effects are enhanced when a nociceptive stimulus is delivered distally (Quevedo and Coghill, 2007) and 
near a joint (Cholewiak and Collins, 2003), as would have uniquely occurred in Experiment 1. Therefore it remains unclear whether the observed effects in Experiment 1 are due to the actual distal location on the forearm and/or whether the nature of the experimental paradigm influenced the results. To answer these questions, we undertook a third experiment.

\section{Experiment 3}

Experiment 3 aimed to replicate the actual location of zones on the forearm used in Experiment 1, but added an extra distal zone near the wrist. If the results of Experiment 1 were a result of insufficient task difficulty with a two-choice paradigm, we should see pain reduction in the middle zone during Localisation and replication of the results of Experiment

2. If, however, the results of Experiment 1 were due to proximity to the joint (enhanced inhibitory effects), then one would expect both Distal zones to exhibit pain reductions during Localisation (versus No-localisation).

\section{Method}

Study design

Randomised repeated measures experiment, involving two conditions separated by 45 minutes, with the order of conditions randomised and counterbalanced.

\section{Participants}

A convenience sample of 18 right-handed, healthy volunteers (10 female) participated. Eligibility criteria were identical to Experiments 1 and 2. All of the participants provided their written informed consent and the study was approved by the Institutional Ethics Committee and was in accordance with the Helsinki Declaration of 1975, as revised in 1983. 
Nociceptive stimuli and experimental procedure

Experiment 3 replicated the protocol of Experiment 1 with the following key difference: another distal zone was added closer to the wrist (i.e., a total of 4 zones: 'proximal', 'middle', 'distal 1', 'distal 2'; see Fig. 2). Thus, in the Localisation condition, the participants were instructed: "You will receive laser stimuli to your forearm. These stimuli will be given to the proximal, distal 1 and distal 2 zone. For each laser stimulus, your task is to tell us in which zone, proximal, distal 1, or distal 2, you received the stimulus.”

Analysis

We performed a 2 x 4 repeated measures analysis of variance (ANOVA) on Pain with two within-participants factors: Condition (Localisation or No-localisation) and Zone (Proximal, Middle, Distal 1, or Distal 2). Where appropriate, post-hoc paired t-tests were used for specific comparisons. Due to the preliminary and exploratory nature of this experiment, we used data from Experiment 1 to power this experiment which suggested that 18 participants were sufficient to detect an effect size of 0.53 , assuming $80 \%$ power and an alpha of 0.05 .

\section{Results}

A total of 20 people were screened for participation; of these, two were left-handed and were excluded.

\section{Pain ratings.}

There was no difference in pain ratings between Conditions $\left(\mathrm{F}_{1,16}=1.08, \mathrm{p}=0.34\right.$, partial $\eta^{2}=$ 0.063 ) and, again, there was a proximal-to-distal gradient in pain (main effect of zone: $F_{3,48}=$ $22.18, \mathrm{p}<0.001$, partial $\left.\eta^{2}=0.58\right)$. Proximal zone pain ratings were significantly larger than those of the middle ( $\mathrm{p}=0.002$ ), Distal 1 , and Distal 2 zones (both $\mathrm{p}<0.001$ ); middle zone pain 
ratings were significantly larger than those of the Distal $1(\mathrm{p}<0.001)$ and Distal 2 zones $(\mathrm{p}=0.001)$, but there was no difference in pain ratings between the Distal 1 and 2 zones $(p=0.10)$. Post hoc tests, considering the effect of zone separately for each condition, support this main effect (see results S1).

The ANOVA also revealed a significant Condition $x$ Zone interaction $\left(F_{3,48}=2.95, p=0.042\right.$, partial $\eta^{2}=0.16$ ). This experiment revealed that the closer to the wrist, the larger the decrease in pain during Localisation, as compared to No-localisation (see Table 1, Fig. 2). That is, pain ratings were lower during Localisation than during No-localisation, only in the most distal zone (Distal zone 2, $\mathrm{t}_{1,17}=2.2, \mathrm{~d}=0.51, \mathrm{p}=0.046$ ); there was no difference between conditions in distal zone 1 ( $\left.\mathrm{t}_{1,17}=1.9, \mathrm{~d}=0.45, \mathrm{p}=0.073\right)$, in the unknown middle zone $\left(\mathrm{t}_{1,17}=0.50, \mathrm{~d}=0.12\right.$, $\mathrm{p}=0.63)$, or the proximal zone $\left(\mathrm{t}_{1,16}=-0.17, \mathrm{~d}=0.04, \mathrm{p}=0.87\right)$.

\section{Experiment 3 Discussion}

The findings of Experiment 3 suggest that the relative location in relation to the wrist joint appears important in the analgesic effect. The lack of middle zone analgesia during Localisation argues against task difficulty exerting an effect and provides support for the view that the actual location of nociceptive stimulus delivery on the arm is important. The lack of significant analgesia during Localisation (compared with No-localisation) in Distal zone 1 (i.e., replication of Experiment 1) suggests that it may not be merely nociceptive afferent density that explains the pain reduction when expectation is spatially divided; rather that there is likely an interaction between expectancy effects, physical nociceptive features inherent to actual stimuli location, and perceptual features related to relative stimuli location. However, the magnitude of pain reduction in Distal zone 1 during Localisation (versus Nolocalisation; non-significant) was very similar to that which occurred for Distal zone 2 but for 
which the analgesic effect was statistically significant, raising the possibility that this former comparison may be underpowered.

\section{Discussion and Conclusions}

We aimed to determine whether expecting nociceptive stimuli in particular skin areas imparted spatially-dependent modulation of pain. We hypothesised that when nociceptive stimuli are expected in two nearby skin areas, there would be an analgesic effect imparted to stimuli delivered in the area between them. This hypothesis was only supported in Experiment 2. Experiments 1 and 3 revealed that proximity to the wrist joint modulated pain during Localisation. In all of the experiments, a consistent effect of actual stimulus location on pain occurred: a proximal-to-distal pain gradient exists on the dorsal forearm. Together, this suggests that pain modulation appears dependent on both the expected location, and the actual location, of the nociceptive stimulus and likely involves multiple mechanisms.

\section{Proximal-to-distal pain gradient of nociceptive sensitivity}

Identical stimuli evoked more pain if they were delivered proximally than if they were delivered distally, which likely reflects the proximal-to-distal gradient of human intraepidermal nerve fibre density (Johansson et al., 1999; Lauria et al., 1999; McArthur et al., 1998), and the positive correlation between thresholds for laser-evoked pinprick sensations and the stimulation site's distance from the brain (Agostino et al., 2000). Although baseline skin temperature appeared to follow a proximal-distal pattern, it did not contribute to the effect on pain (pain results unchanged when controlling for temperature).

\section{Spatial effects on pain during Localisation: Task-dependent dynamic tuning}

That dorsal horn RFs can be 'focussed' - i.e., via task-dependent dynamic tuning (Compte and Wang, 2006; Hayes et al., 1981) - by dividing attention (Quevedo and Coghill, 2007), 
led us to predict that middle zone stimuli would evoke less pain during Localisation than stimuli to the outer zones. Attentional-related changes (Compte and Wang, 2006; Hayes et al., 1981), including increased surround inhibition and decreased RF size (Compte and Wang, 2006; Quevedo and Coghill, 2007), are thought to mediate this focussing of neural resources. We postulated that such an effect would create a 'silent zone' between the two expected locations, resulting in decreased pain (Quevedo and Coghill, 2007): consistent with the results of Experiment 2.

Dynamic tuning of RFs occurs via the descending nociceptive modulation system, including the anterior cingulate cortex, amygdala, periaqueductal grey, rostral ventromedial medulla, and dorsal horn neurons (Basbaum and Fields, 1984; Bingel and Tracey, 2008). Coupling of cingulo-frontal regions with subcortical areas of the descending modulatory system has been confirmed for attentional modulation of pain (Valet et al., 2004). Expectation-driven analgesia has been demonstrated between body segments (analgesia in one limb but not another; Benedetti et al., 1999) and within a body segment (Eippert et al., 2009). Such findings suggest that descending control could exert the observed effects.

The conceptual model of task-dependent dynamic tuning may have relevance for interpreting Experiments 1 and 3. If RF size reduces with focussed attention to an area, areas with lower innervation density (distal relative to proximal) should be more affected, demonstrating a larger overall inhibitory effect. Lower pain during Localisation in only the most distal zone (Experiments 1 and 3) is consistent with findings from divided attention paradigms: the inhibitory effect on pain is strongest for distal stimuli (Quevedo and Coghill, 2007). Proximity to the distal area of the forearm and wrist joint appears essential: in Experiment 2 (all zones located more proximally on the forearm) the distal zone had no pain decrease during Localisation. 
The exact mechanism for analgesia based on proximity to the wrist joint (Experiments 1 and 3), and specific to Localisation, is unclear. Anatomical points, such as joints, are suggested to provide a perceptual anchor with which to identify a stimulation site (Culver, 1970). Psychophysical studies suggest that a distal localisation bias exists for the forearm: stimuli are perceived to occur closer to the wrist than they actually are (Azanon et al., 2010) (although individual-specific differences may exist for thermal stimuli; Trojan et al., 2006). The data from Experiment 1 are consistent with this bias - the perceived location of stimulation was distal to the actual location (all zones) for most participants. On the contrary, Experiment 2 data showed more accurate (proximal zone) or varied (distal zone) perceptions of zone location. Perhaps this differential perceptual shift, combined with increased attentioninduced inhibition in distal locations, compounded the effect on the distal zone.

\section{$\underline{\text { Interaction between pain localisation and intensity modulation }}$}

Nociceptive spatial acuity is relevant in the modulation of pain: knowing the location of a stimulus can decrease its threat value, and thus, decrease pain (Johnston et al., 2012). Spatial acuity for nociceptive stimuli on the hairy skin is greatest in proximal limb segments than in distal (Mancini et al., 2014); whether variation in acuity occurs within a body segment is unknown. Increased tactile acuity exists over the joints (Cholewiak and Collins, 2003; Cody et al., 2008) suggesting that nociceptive acuity could also be heightened over the joints (mediated by the convergence of tactile and nociceptive pathways Haggard et al., 2013; Kenshalo et al., 2000; Maixner et al., 1986; Mancini et al., 2012). While localisation errors had similar patterns for proximal and distal zones in Experiments 1 and 2 (errors lower in proximal zones, higher in distal), overall localisation errors were lower in Experiment 1 than in Experiment 2 (zones more proximal) which may have implications for bottom-up mechanisms, as discussed below. 
Cortico-cortical interactions may be implicated in the zone-specific analgesia when nociceptive localisation judgements occur. Cortical activity differs between tasks that involve attending to the location versus the intensity of nociceptive stimuli (Kulkarni et al., 2005; Lobanov et al., 2013). That fine-grained nociceptive maps exist in the primary somatosensory cortex (S1; Mancini et al., 2012) suggests that spatially-precise modulation of nociceptive information is possible. Attentional state may modulate S1 activity - S1 blood flow is higher when subjects attend to a tactile stimulus than when they attend elsewhere (Meyer et al., 1991). Attentional state also modulates activity of the insula (Bantick et al., 2002), an area with demonstrated somatotopic organisation for nociceptive stimuli (Mazzola et al., 2009). Perhaps expectancy-induced, attentional focusing could also modulate S1/insular activity in a precise manner, which may then alter pain.

\section{Bottom-up effects of attention and pain modulation}

The lack of middle zone analgesia during the Localisation condition of Experiments 1 and 3 might also be related to bottom-up effects on spatial attention. Nociceptive ERPs are larger when the stimuli are novel in timing or spatial location than when they are not (Legrain et al., 2003; Legrain et al., 2002; Legrain et al., 2009), regardless of the focus of attention(Legrain et al., 2002). Perhaps, then, decreased spatial judgment precision in Experiment 2 (increased overall localisation errors) resulted in the inability to detect 'spatial novelty' at a systems level, allowing for analgesic effects in the middle zone during divided expectation.

\section{$\underline{\text { Limitations }}$}

We cannot exclude the possibility that the mirror used to provide an illusory visual image of the hidden arm may have induced a ventriloquist effect (visual information informs one's perception of where a non-visual stimulus originates from) such that it did not feel that the stimuli were occurring on the real arm. Tactile stimuli that are 'ventriloquized' are perceived 
as less intense than those that are not (Walton and Spence, 2004), but such an effect would not be zone-specific, making it unlikely here.

Use of identical nociceptive stimulus energy for all zones resulted in unmatched pain intensity ratings between zones. This is unlikely to have impacted our results for two reasons: (i) the proximal-to-distal pain gradient occurred in all experiments and despite this, different zone-specific analgesic effects occurred; (ii) Experiment 2 did not demonstrate analgesia for the zone with the lowest pain score (distal zone), thus arguing against an effect of attention, dependent on the reported pain intensity, which may be predicted by attentional models (Eccleston and Crombez, 1999; Legrain et al., 2012). It is possible that generally low pain ratings could create a floor effect: similar results in Experiments 1 and 3, despite overall differences in pain intensity, suggest that the magnitude of pain was not of primary importance.

There are also some task-specific considerations: participants attributed middle zone stimuli to the proximal zone more often than to the distal zone, raising the possibility that they associated the proximal zone with pain more than the distal zone. It is unclear whether participants used varied strategies (e.g., attending towards or away from certain expected stimuli zones) in order to localise stimuli. Consistent results in Experiments 1 and 3, despite different groups of participant, support the presence of broadly replicable localisation strategies. Future study, including phenomenological assessment of localisation strategy, seems warranted.

\section{Implications:}

Our results demonstrate spatial precision of pain modulation when expectancy of spatial location of a stimulus is manipulated, suggestive of a connection between somatotopic body maps and nociceptive modulation. While the expectation-driven modulation of pain can have 
spatially-specific effects, these appear to be influenced by both a proximal-distal gradient of nociceptive sensitivity (possibly reflective of nociceptor afferent density), and by proximity to a distal joint. Dynamic spatial tuning seems to be more precise, and the mechanisms underpinning it more varied, than previously thought. Our results raise the possibility of clinical applications - perhaps capturing several mechanisms will lead to ideal locations and expectation manipulations with which to optimise analgesia.

\section{Acknowledgements:}

We would also like to acknowledge the following people for their assistance with the study: Miss Sarah Wallwork for assistance in analysing temperature data; Dr Joerg Trojan for relevant discussions regarding interpretation of the findings; and Dr Giandomenico Ianetti for his assistance with the data analysis.

\section{Author contributions:}

All authors have read and approved of this manuscript. Tasha Stanton, Lorimer Moseley, Charles Spence and Flavia Mancini provided concept/idea/study design. Tasha Stanton, Helen Gilpin and Emily Reid performed data collection. Tasha Stanton and Lorimer Moseley provided equipment. Lorimer Moseley and Charles Spence provided fund procurement (National Health and Medical Research Council Project Grant; no role was played by this funding source in study procedures, manuscript preparation or submission). Tasha Stanton, Lorimer Moseley and Helen Gilpin were involved in data analysis. All authors were involved in the writing of the manuscript, discussed the results and all provided consultation (including review of the manuscript prior to submission). 


\section{References:}

Agostino, R., Cruccu, G., Iannetti, G., Romaniello, A., Truini, A., and Manfredi, M. (2000). Topographical distribution of pinprick and warmth thresholds to $\mathrm{CO}_{2}$ laser stimulation on the human skin. Neurosci Lett 285, 115-118.

Azanon, E., Longo, M.R., Soto-Faraco, S., and Haggard, P. (2010). The posterior parietal cortex remaps touch into external space. Curr Biol 20, 1304-1309.

Bantick, S.J., Wise, R.G., Ploghaus, A., Clare, S., Smith, S.M., and Tracey, I. (2002). Imaging how attention modulates pain in humans using functional MRI. Brain 125, 310-319. Basbaum, A.I., and Fields, H.L. (1984). Endogenous pain control systems: brainstem spinal pathways and endorphin circuitry. Annu Rev Neurosci 7, 309-338.

Benedetti, F., Arduino, C., and Amanzio, M. (1999). Somatotopic activation of opioid systems by target-directed expectations of analgesia. J Neurosci 19, 3639-3648.

Bingel, U., and Tracey, I. (2008). Imaging CNS modulation of pain in humans. Physiol 23, 371-380.

Cholewiak, R.W., and Collins, A.A. (2003). Vibrotactile localization on the arm: Effects of place, space and age. Percept Psychophys 65, 1058-1077.

Cody, F.W.J., Garside, R.A.D., Lloyd, D., and Poliakoff, E. (2008). Tactile spatial acuity varies with site and axis in the human upper limb. Neurosci Lett 433, 103-108.

Compte, A., and Wang, X.J. (2006). Tuning curve shift by attention modulation in cortical neurons: a computational study of its mechanisms. Cereb Cortex 16, 761-778.

Culver, C.M. (1970). Errors in tactile localization. Am J Psychol 83, 420-427. Eccleston, C., and Crombez, G. (1999). Pain demands attention: A cognitive-affective model of the interruptive function of pain. Psychol Bull 125, 356-366. 
Egsgaard, L.L., Petrini, L., Christoffersen, G., and Arendt-Nielsen, L. (2011). Cortical responses to the mirror box illusion: a high-resolution EEG study. Exp Brain Res 215, 345357.

Eippert, F., Finsterbusch, J., Bingel, U., and Buchel, C. (2009). Direct evidence for spinal cord involvement in placebo analgesia. Science 326, 404.

Faggin, B.M., Nguyen, K.T., and Nicolelis, M.A. (1997). Immediate and simultaneous sensory reorganization at cortical and subcortical levels of the somatosensory system. Proc Natl Acad Sci USA 94, 9428-9433.

Faul, F., Erdfelder, E., Lang, A.G., and Buchner, A. (2007). G*Power 3: A flexible statistical power analysis program for the social, behavioral, and biomedical sciences. . Behavior Research Methods 39, 175-191.

Haggard, P., Iannetti, G.D., and Longo, M.R. (2013). Spatial sensory organization and body representation in pain perception. Curr Biol 23, R164-R176.

Hayes, R.L., Dubner, R., and Hoffman, D.S. (1981). Neuronal activity in medullary dorsal horn of awake monkeys trained in a thermal discrimination tasks. II. Behavioural modulation of responses to thermal and mechanical stimuli. J Neurophysiol 46, 428-443.

Iannetti, G.D., Hughes, N.P., Lee, M.C., and Mouraux, A. (2008). Determinants of Laserevoked EEG reponses: Pain perception or stimulus saliency. J Neurophysiol 100, 815-828. Iannetti, G.D., Zambreanu, L., and Tracey, I. (2006). Similar nociceptive afferents mediate psychophysical and electrophysiological responses to heat stimulation of glabrous and hairy skin in humans. J Physiol 577, 235-248.

Johansson, O., Wang, L., Hilliges, M., and Liang, Y. (1999). Intraepidermal nerves in the human skin: PGP 9.5 immunohistochemistry with special reference to the nerve density in skin from different body regions. J Peripher Nerv Syst 4, 43-52. 
Johnston, N.E., Atlas, L.Y., and Wagner, T.D. (2012). Opposing effects of expectancy and somatic focus on pain. PLoS ONE 7, e38854.

Kennett, S., Taylor-Clarke, M., and Haggard, P. (2001). Noninformative vision improves the spatial resolution of touch in humans. Curr Biol 7, 1188-1191.

Kenshalo, D.R., Iwata, K., Sholas, M., and Thomas, D.A. (2000). Response properties and organization of nociceptive neurons in area 1 of monkey primary somatosensory cortex. $\mathrm{J}$ Neurophysiol 84, 719-729.

Koyama, T., McHaffie, J.G., Laurienti, P.J., and Coghill, R.C. (2005). The subjective experience of pain: where expectations become reality. Proc Natl Acad Sci USA 102, 12950 12955.

Kulkarni, B., Bentley, D.E., Elliot, R., Youell, P., Watson, A., Derbyshire, S.W.G., Frackowiak, R.S.J., Friston, K.J., and Jones, A.K.P. (2005). Attention to pain localization and unpleasantness discriminations the functions of the medial and lateral pain systems. Eur $\mathrm{J}$ Neurosci 21, 3133-3142.

Lauria, G., Holland, N., Hauer, P., Cornblath, D.R., Griffin, J.W., and McArthur, J.C. (1999). Epidermal innervation: changes with aging, topographic location, and in sensory neuropathy. J Neurol Sci 164, 172-178.

Legrain, V., Geurit, J.M., Bruyer, R., and Plaghki, L. (2003). Electrophysiolgical correlates of attentional orientation in humans to strong intensity deviant nociceptive stimuli, inside and outside the focus of spatial attention. Neurosci Lett 339, 107-110.

Legrain, V., Guerit, J.-M., Bruyer, R., and Plaghki, L. (2002). Attentional modulation of the nociceptive processing into the human brain: selective spatial attention, probability of stimulus occurrence and target detection effects on laser evoked potentials. Pain 99, 21-39. 
Legrain, V., Mancini, F., Sambo, C.F., Torta, D.M., Ronga, I., and Valentini, E. (2012). Cognitive aspects of nociception and pain: briding neurophysiology with cognitive psychology. Neurophysiol Clin 42, 323-335.

Legrain, V., Perchet, C., and Garcio-Larrea, L. (2009). Involuntary orienting of attention to pain. J Neurophysiol 102, 2423-2434.

Lobanov, O.V., Quevedo, A.S., Hadsel, M.S., Kraft, R.A., and Coghill, R.C. (2013). Frontoparietal mechanisms supporting attention to location and intensity of painful stimuli. Pain 154, 1758-1768.

Maixner, W., Dubner, R., Bushnell, M.C., Kenshalo, D.R.J., and Oliveras, J.L. (1986). Widedynamic-range dorsal horn neurons participate in the encoding process by which monkeys perceive the intensity of noxious heat stimuli. Brain Res 374, 385-388.

Mancini, F., Bauleo, A., Cole, J., Lui, F., Porro, C.A., Haggard, P., and Iannetti, G.D. (2014). Whole-body mapping of spatial acuity for pain and touch. Ann Neurol 75, 917-924.

Mancini, F., Haggard, P., Iannetti, G.D., Longo, M.R., and Sereno, M.I. (2012). Fine-grained nociceptive maps in primary somatosensory cortex. J Neurosci 32, 17155-17162.

Mazzola, L., Isnard, J., Peyron, R., Guenot, M., and Mauguiere, F. (2009). Somatotopic organization of pain responses to direct electrical stimulation of the human insular cortex. Pain 146, 99-104.

McArthur, J.C., Stocks, E.A., Hauer, P., Cornblath, D.R., and Griffin, J.W. (1998). Epidermal nerve fiber density: normative reference range and diagnostic efficiency. Arch Neurol 55, $1513-1520$.

McCaul, K.D., and Haugtvedt, C. (1982). Attention, distraction, and cold-pressor pain. J Pers Soc Psychol 43, 154-162. 
Meyer, E., Ferguson, S.G., Zatorre, R.J., Alivisatos, B., Marrett, S., Evans, A.C., and Hakim, A.M. (1991). Attention modulates somatosensory cerebral blood flow response to vibrotactile stimulation as measured by positron emission tomography. Ann Neurol 29, 440-443.

Morch, C.D., Andersen, O.K., Quevedo, A.S., Arendt-Nielsen, L., and Coghill, R.C. (2010). Exteroceptive aspects of nociception: Insights from graphesthesia and two-point discrimination. Pain 151, 45-52.

Morgan, M.M., and Whitney, P.K. (1996). Behavioral analysis of diffuse noxious inhibitory controls (DNIC): Antinociception and escape reactions. Pain 66, 307-312.

Moseley, G.L., Olthof, N., Venema, A., Don, S., Mijers, M., Gallace, A., and Spence, C. (2008). Psychologically induced cooling of a specific body part caused by the illusory ownership of an artificial counterpart. Proc Natl Acad Sci USA 105, 13169-13173. Quevedo, A.S., and Coghill, R.C. (2007). Attentional modulation of spatial integration of pain: Evidence for dynamic spatial tuning. J Neurosci 27, 11635-11640.

Romero, Y.R., Straube, T., Nitsch, A., Miltner, W.H.R., and Weiss, T. (2013). Interaction between stimulus intensity and perceptual load in the attentional control of pain. Pain 154, 135-140.

Trojan, J., Kleinbohl, D., Stolle, A.M., Andersen, O.K., Holzl, R., and Arendt-Nielsen, L. (2006). Psychophysical 'perceptual maps' of heat and pain sensations by direct localization of $\mathrm{Co}_{2}$ laser stimuli on the skin. Brain Res 1120, 106-113.

Valet, M., Sprenger, T., Boecker, H., Willoch, F., Rummeny, E., Conrad, B., Erhard, P., and Tolle, T.R. (2004). Distraction modulates connectivity of the cingulofrontal cortex and the midbrain during pain: an fMRI analysis. Pain 109, 399-408.

Van Damme, S., Gallace, A., Spence, C., Crombez, G., and Moseley, G.L. (2009). Does the sight of physical threat induce a tactile processing bias? Modality-specific attentional facilitation induced by viewing threatening pictures. Brain Res 1253, 100-106. 
Van Ryckeghem, D.M.L., Van Damme, S., Crombez, G., Eccleston, C., Verhoeven, K., and Legrain, V. (2011). The role of spatial attention in attentional control over pain: an experimental investigation. Exp Brain Res 208, 269-275.

Walton, M., and Spence, C. (2004). Cross-modal congruency and visual capture in a visual elevation discrimination task. Exp Brain Res 154, 113-120. 


\section{Figure Titles and Legends:}

Fig. 1. Experimental set-up.

Figure legend: Physical set-up of participant and examiner. The participant watches the reflection of his/her left arm in a mirror that is aligned with the opaque cloth.

Fig. 2. Pain intensity ratings for Experiments 1-3 with sites of laser pulse application for the Localisation condition.

Figure legend: Mean pain ratings (SEM) for each zone and condition. In all studies there was a main effect of zone ( $\mathrm{p}<0.001$ for all) and a significant interaction between zone and condition (E1: $\mathrm{p}=0.02$; E2: $\mathrm{p}=0.04$, E3: $\mathrm{p}=0.04)$. Post hoc tests: ${ }^{\wedge} \mathrm{p}<0.05$; Pairwise comparisons: ${ }^{*} \mathrm{p}<0.01 ;{ }^{* *} \mathrm{p}<0.001$. The white perpendicular lines were drawn on the participant's arms during the Localisation condition. The dotted white lines convey the approximate location of the middle zone on the forearm; in reality these lines were not drawn on participants’ arms. In the No-localisation condition, laser stimuli were provided to identical areas of the arm, but without any skin marking.

\section{Table captions and legends:}

Table 1 - Participant characteristics and pain ratings (separated by Zone and Condition) for Experiments 1, 2 and 3.

Legend: Participant age is presented as mean (standard deviation). Pain ratings presented as means (95\% confidence intervals). N/A = not applicable. ${ }^{*}=$ significant reduction in pain rating during Localisation condition compared with No-Localisation condition in the zone $(\mathrm{p}<0.05)$.

Table 2. Arm temperature results specific to zone and condition for Experiments 1 and 2. 
Legend: Temperature measured in degrees Celsius. $\Delta \mathrm{T}$ represents change in temperature (post-testing minus pre-testing temperature). All values are means (SD).

Table 3. Error rates and perceptual data for Experiments 1 and 2.

Legend: Localisation error was calculated by summing the number of errors for each participant (over the specified pulse range) and dividing by the number of participants. *Missed indicating this zone 